\title{
Mujeres migrantes latinoamericanas en trabajos masculinizados en Santiago: reconocimiento e interseccionalidad*
}

\author{
Latin American migrant women in masculinised jobs in Santiago: \\ Recognition and intersectionality
}

\author{
María Elvira Cárdenas ${ }^{* *}$ \\ Pamela Caro Molina*** \\ Centro de Estudios en Familia, Trabajo y Ciudadanía (CIELO), Facultad de Ciencias \\ Sociales y Comunicaciones, Universidad Santo Tomás, Santiago, Chile.
}

\begin{abstract}
* El presente artículo es producto del proyecto Anid/Fondecyt/Regular $\mathrm{N}^{\circ} 1180590$ 2018-2020, por lo que agradecemos a Anid/Fondecyt. Sus resultados emergen de la tesis de magíster en Trabajo Social de la Pontificia Universidad Católica de Chile realizada en el marco del proyecto citado.

*** Magíster en Trabajo Social, Pontificia Universidad Católica de Chile. Magíster en Seguridad y Defensa Nacionales, Escuela Superior de Guerra "General Rafael Reyes Prieto". Socióloga, Universidad Nacional de Colombia. Asistente de investigación en el Centro de Investigación y Estudios en Familia, Trabajo y Ciudadanía (CIELO), Universidad Santo Tomás. Dirección: Av. Ejército Libertador 146, Santiago, Región Metropolitana. Correo electrónico: mariaelvira.cardenas@gmail.com ORCID: https://orcid.org/0000-0002-7972-3409

**** Doctora en Estudios Americanos, Instituto de Estudios Avanzados, Universidad de Santiago de Chile. Magíster en Ciencias Sociales, ILADES/Universidad Gregoriana de Roma. Licenciada en Trabajo Social y asistente social, Pontificia Universidad Católica de Chile. Directora del Centro de Investigación y Estudios en Familia, Trabajo y Ciudadanía (CIELO), Universidad Santo Tomás. Dirección: Av. Ejército Libertador 146, Santiago, Región Metropolitana. Correo electrónico: pamelacaro1@ santotomas.cl ORCID: https://orcid.org/0000-0001-8177-9295
\end{abstract}

Cómo citar este artículo: Caro M., P., y Cárdenas, María E. (2020). Mujeres migrantes latinoamericanas en trabajos masculinizados en Santiago: reconocimiento e interseccionalidad. Si Somos Americanos. Revista de Estudios Transfronterizos, 21(1), 103-128. doi: 10.4067/S0719-09482021000100103 


\title{
Resumen
}

El artículo aborda la forma en que las mujeres migrantes, que trabajan en empleos masculinizados en Santiago, experimentan las relaciones e interacciones sociales. Se analizan estas desde las nociones de justicia de género y reconocimiento en sus entornos laborales, considerando la teoría del reconocimiento de Axel Honneth y la discusión teórica sobre interseccionalidad desde la perspectiva anglo y latinoamericana (Galaz, Pavez, Álvarez y Hedrera, 2019; Troncoso, Follegati y Stutzin, 2019; Espinoza, 2016; Curiel, 2014; Lugones, 2005 y Crenshaw, 1989). La metodología cualitativa empleada en esta investigación se basó en la realización de entrevistas en profundidad a dichas mujeres. Como principal resultado, se tiene que las migrantes experimentan injusticias de género relacionadas con la valoración cultural de sus capacidades específicas de éxito otorgadas en entornos laborales masculinizados. Asimismo, se concluye que esta injusticia de género que se presenta en los trabajos masculinizados se experimenta de una manera distinta cuando se intersectan otros ejes de opresión (como la nacionalidad o la clase social), desde los cuales no solo se evidencia la falta de reconocimiento, sino también cómo operan las herramientas de resistencia generadas por las mujeres migrantes.

Palabras clave: migración, género, interseccionalidad.

\begin{abstract}
This article analyses how migrant women who work in masculinised jobs in Santiago experience social relationships and interactions. Its focus is gender justice and women's recognition within the work environment based on Axel Honneth's Theory of Recognition and the theoretical discussion of intersectionality from an Anglo and Latin American perspective (Galaz et al., 2019; Troncoso et al., 2019; Espinoza, 2016; Curiel, 2014; Lugones, 2005 and Crenshaw, 1989). The qualitative methodology is based on indepth interviews with some of the women in question. The main result of the study showed that migrant women experience gender injustices related to the cultural appraisal of their specific capacities for success offered to them in masculinised work environments. The type of gender injustice that occurs in masculinised jobs is experienced differently when other forms of oppression intersect (such as nationality or social class). We observed both a lack of recognition and how the tools of resistance generated by migrant women operate.
\end{abstract}

Keywords: Migration, gender, intersectionality. 


\section{Introducción}

En los últimos años se ha presentado una cifra sin precedentes en cuanto a la participación de mujeres en el mercado laboral en América Latina, llegando por primera vez en 2017 a superar la barrera de1 50\%: 50,2\%. Por su parte, en Chile, la tasa para ese mismo año fue de 48,3\% (OIT, 2017). A pesar de esto, se siguen manteniendo en el país procesos de desigualdad, discriminación y falta de oportunidades a nivel laboral debido al género, que se ven reflejados en aspectos como el salario (Valdés y Meller, 2014) y la segregación horizontal y vertical de género (Cárdenas, Correa y Pardo, 2014).

A la realidad desigual laboral que viven las mujeres por su condición de género, puede sumarse otra característica, que es la de ser migrante. En Chile ha aumentado la migración en los últimos años, pasando de 45 mil personas al iniciar la década de 1990 (INE, 2018) -momento en que se empezaron a renovar los flujos migratorios hacia Chile (Carrère y Carrère, 2015) - a 1.492.522 al 31 de diciembre de 2019 (INE y DEM, 2020).

Sumado a lo anterior, en los últimos años se ha presentado una feminización en la migración mundial (Acosta, 2012) y en América Latina (Stefoni, 2018), acompañada del aumento de la migración intrarregional, en la que Chile aparece como uno de los destinos más frecuentes y apreciados para las migrantes (Martínez y Orrego, 2016; Lobos, 2017; Rojas y Silva, 2016).

Según Thayer (2012), suele suceder que la población migrante llegue al país de destino a ocupar un segmento específico del mercado de trabajo, y Chile no está fuera de esta realidad. Los/as ciudadanos/as del país rechazan determinados puestos de trabajo por motivos relativos al salario o al estatus, empleos precarios que suelen ser ocupados por migrantes. Esta población tiende a ocupar una posición de subordinación en la estructura ocupacional, inferior a la de los/as ciudadanos/as del país (Thayer, 2012). Lo anterior pone de manifiesto que muchos/as trabajadores/as migrantes no tienen opciones de acceder a trabajos con mejores condiciones laborales, evidenciándose una intersección entre migración y categorías como género que profundizan la estratificación laboral (Stefoni y Stang, 2017).

A partir de tales consideraciones surge la pregunta, ¿en qué se están desempeñando las personas migrantes en Chile? Al respecto, según la encuesta Casen (Ministerio de Desarrollo Social, 2017) casi la mitad se desempeña en la rama de comercio $(44,4 \%)$, seguida del rubro de hoteles y restaurantes $(29,9 \%)$ y en actividades empresariales, inmobiliarias y de alquiler (24,6\%). Al observar la distribución por sexo, y enfocándose en las cifras relativas a mujeres, se pone de manifiesto que la mayoría de las migrantes trabajan en el sector comercio $(21,9 \%)$, seguido de hogares privados con servicio doméstico $(21,5 \%)$ y hoteles y restaurantes $(16 \%)$. Sin embargo, llama la atención que también hay mujeres migrantes que están desempeñándose en ramas tradicionalmente masculinas como el suministro de electricidad, gas y agua $(0,1 \%)$, y el transporte, almacenamiento y comunicaciones $(3,7 \%)$, pero claramente en menor proporción que los primeros rubros, demostrando una segregación ocupacional de género. Entre los 
empleos en ramas masculinizadas, las migrantes se desempeñan tanto en empleos calificados como no calificados, entendiendo estos últimos como los empleos para los cuales no se necesita un proceso educativo técnico o profesional para adquirir las competencias necesarias para su desarrollo (Riquelme, López y Olivares, 2014).

Es importante destacar que las tareas y ocupaciones se han ido sexuando (Vidal, 2017). Como lo plantea Díaz (2014), hay ocupaciones mayoritariamente desempeñadas por hombres y otras por mujeres, cuyos contenidos se asocian a roles sexuales estereotipados, eligiendo así mujeres y hombres ocupaciones asociadas a referentes propios de su sexo o a la imagen de género tradicionalmente instalados por la cultura de manera histórica. Estos reproducen, por un lado, la extensión del rol reproductivo de las mujeres, y por otro, la división de tareas, lo cual pone de manifiesto los privilegios de un sexo posibilitados por la subordinación del otro, donde ha de considerarse la invisibilización del trabajo femenino y su concentración en el ámbito reproductivo y no remunerado (Benería, 2006). Cuando se trata de escoger carreras profesionales, aquellas que eligen las mujeres adquieren menor valor social y son peor remuneradas (Comunidad Mujer, 2016). En adelante se entenderá aquel trabajo desempeñado en su mayoría por hombres como "trabajo masculinizado" y aquel desempeñado en su mayoría por mujeres como "trabajo feminizado".

Revisando la literatura sobre género y migración, Galaz, Becerra, Álvarez y Hedrera (2016) abordan las prácticas y discursos que surgen dentro de ciertos dispositivos de intervención social que trabajan con mujeres migrantes y que han sido implementados en Chile. La mayoría de los trabajos circula en torno a mujeres migrantes que se desempeñan en tareas hegemónicamente feminizadas, como domésticas y de cuidado (Canales, 2014; Salas, 2014; Stefoni y Fernández, 2012), enfatizando en que las migrantes se concentran en empleos menos deseados por las nativas, caracterizados por su baja remuneración y mayor precariedad laboral (Acosta, 2013); y no obstante aquellas tienen una situación migratoria regular, desempeñan tales oficios sin un contrato de trabajo, exponiéndose a largas jornadas laborales y malos tratos (Leiva, Mansilla y Comelin, 2017). Por otra parte, se encontraron estudios relativos a las mujeres que laboran en trabajos masculinizados (Angelcos e Ísola, 2017; Jiménez, Rojas y Troncoso, 2014; Salinas y Romaní, 2016), sin diferenciación específica entre migrantes y no migrantes, aun existiendo un porcentaje de mujeres migrantes que trabajan en dichas ramas, como lo han indicado estudios previos (Stefoni, Leiva y Bonhomme, 2017) con foco en la construcción. En menor proporción existen estudios sobre la participación de mujeres migrantes en empleos calificados, como el de Gissi y Martínez (2018).

El objetivo de este artículo es dar cuenta de la forma en que mujeres migrantes, que trabajan en empleos masculinizados en Santiago de Chile, experimentan las relaciones e interacciones sociales, analizando estas a partir de las nociones de justicia de género y de reconocimiento en sus entornos laborales. El texto se enfoca en ocupaciones masculinizadas relativas a áreas de ingeniería/tecnología, servicio de seguridad y gasolineras. Las preguntas que guían este estudio indagan en las condiciones laborales de las migrantes que se desempeñan en empleos masculinizados, las características de 
su inserción, las dificultades que atraviesan y las valoraciones que hacen de su actividad, considerando una perspectiva de género y aspectos de interseccionalidad.

Teniendo a la vista las condiciones específicas de las mujeres en el entorno laboral chileno y ponderando la relevancia por evidenciar el trabajo de mujeres migrantes en contextos masculinizados, el artículo parte de la hipótesis de que las migrantes que trabajan en empleos masculinizados en Santiago de Chile viven injusticias de género, las que se entrecruzan con injusticias derivadas de su nacionalidad y de clase social, que se manifiestan en conflictos ligados a la falta de reconocimiento en sus entornos laborales.

\section{Migración y género desde la mirada del reconocimiento}

El estudio base de este artículo (Cárdenas, inédita) se guio conceptualmente bajo el marco de la teoría del reconocimiento de Honneth (2006) y de la propuesta de Thayer (2013), lo que permitió abordar aspectos relativos al salario, la estima social y la autovaloración de mujeres migrantes insertas en empleos y rubros de trabajo masculinizados. De igual manera, se abordó la interseccionalidad (Crenshaw, 1989; Curiel, 2014; Espinoza, 2016; Galaz et al., 2019; Lugones, 2005; Troncoso et al., 2019), como lineamiento conceptual para tratar los diferentes ejes de diferenciación que pudiesen o no posibilitar el reconocimiento.

Los postulados de Thayer (2013) y Honneth (2006) se utilizan para plantear teóricamente el fenómeno de la participación de mujeres migrantes en empleos masculinizados, aportando a la discusión académica que han entregado estudios existentes sobre los procesos y consecuencias de la migración en Chile (Pavez-Soto y Galaz, 2018; Stefoni, 2014; Suárez-Cabrera, 2015; Tijoux y Palominos, 2015).

Honneth (2006) distingue tres formas de relación que se deslindan por sus principios normativos internos $\mathrm{y}$ tienen distintas formas de reconocimiento mutuo. Se problematizan tres esferas diferenciadas que representan perspectivas normativas ligadas a quienes pueden discutir razonablemente que las formas de reconocimiento no son adecuadas o no son suficientes. Estas son el amor, la igualdad jurídica y el principio del éxito. En esta última nos centramos en este artículo, la que se relaciona con el aporte social que se estima hace un sujeto a la sociedad. Al respecto, en las luchas en búsqueda del reconocimiento, los sujetos promueven capacidades y actividades que no se valoran, por lo que acuden al principio del éxito buscando una mayor estima social y una redistribución de recursos materiales (Honneth, 2006), interpretando las desigualdades materiales vinculadas a la distribución como formas de transgresión de reivindicaciones bien fundamentadas de reconocimiento. Se presenta la distribución de acuerdo con principios de valor relacionados con el respeto y la estima social (Honneth, 2006), lo que hace referencia a que el valor dado a cada profesión u oficio tiene una relación con la distribución de bienes materiales, poniendo de relieve la presencia de valores culturales en la consolidación institucional de la esfera económica y las reglas de 
remuneración generadas en torno a consideraciones de eficiencia ligadas a perspectivas culturales (Honneth, 2006).

Desde su propuesta, la injusticia dice relación con la negación de reconocimiento, determinado por las capacidades que los sujetos afectados consideran como no reconocidas sin justificación; de tal forma, la justicia correspondería a obtener el reconocimiento legítimo, que equivaldría a lo observado como aplicación justa del reconocimiento del principio del éxito (Honneth, 2006).

Por su parte, la categoría de género, entendida como una medida cultural determinante de la estima social reflejada en una actividad concreta (Honneth, 2006), ha sido abordada por distintos/as autores/as (Bourdieu, 2000; Butler, 2011; Pateman, 1988; Scott, 1986), quienes coinciden en que se trata de una categoría analítica que permite entender las relaciones entre hombres y mujeres como relaciones de poder, en las que los roles de género son una construcción cultural e histórica. Hay consenso en que su principal transmisión ocurre a través de la socialización, que solidifica percepciones y estereotipos tradicionalmente conformados para interpretar las prácticas y narrativas de hombres y mujeres en cada sociedad. Fraser (2006) la vincula al ámbito del reconocimiento, sosteniendo que el género hace referencia a una diferenciación social bidimensional, una categoría híbrida involucrada en la estructura económica y en el estatus de la sociedad de forma simultánea. Desde la mirada de la redistribución, el género puede abordarse como principio organizador de la estructura económica de la sociedad, mientras que, desde la mirada del reconocimiento, el género codifica patrones culturales de interpretación en el estatus social.

Honneth (2006), partiendo de la idea de que la valoración cultural de las capacidades específicas de éxito lleva a la demarcación social de las profesiones, aborda la relación entre reconocimiento y género evidenciando la existencia de prejuicios sobre los límites de las capacidades de las mujeres en la construcción social de los campos laborales y profesionales. Según el autor, en una jerarquía de estatus social, las actividades laborales predominantemente practicadas por mujeres tenderán a caer y las practicadas por hombres tenderán a subir en prestigio y reconocimiento, explicando la infravaloración de las primeras. Lo anterior ubica al género como medida cultural que establece la estima social evidenciada en una actividad concreta, al considerarla como masculina o femenina, mostrando la forma en que los puntos de vista culturales de evaluación sobre el aporte de distintos grupos a la reproducción social tienen influencia en la legitimación del orden de la distribución social, determinando el punto hasta el que cada actividad profesional recibe reconocimiento social (Honneth, 2006). En este sentido, los conflictos se generan cuando los grupos sociales cuestionan esas formas de clasificación, esperando mayor estima en lo relativo a sus aportes sociales y a la redistribución económica (Honneth, 2006).

En consecuencia, se entenderá injusticia de género como la falta de reconocimiento en razón del género, asociando "desigualdades de género" con "injusticia de género", al considerar que las primeras subyacen a la falta de reconocimiento de las capacidades de las mujeres para el principio del éxito. 
Siguiendo la línea de Honneth (2006), se examina la migración desde la perspectiva del reconocimiento, recogido por Thayer (2013), al decir que la imagen de la condición de los/as migrantes, junto con sus estrategias de incorporación, se construyen en gran medida a partir del reconocimiento otorgado por el Estado y la sociedad receptora. Considerando tres dimensiones en las que pueden enmarcarse las relaciones de reconocimiento (las relaciones primarias, el acceso igualitario al espacio público y la valoración de la contribución de cada sujeto a la reproducción material y simbólica), este autor propone que desde allí se generan, para cada una de las dimensiones, respectivamente, y reflexionando sobre la imagen que los sujetos elaboran de sí mismos, la autoconfianza, el autorrespeto y la autovaloración. Esta última se fundamenta en la consideración social de la contribución de los sujetos en la reproducción material y simbólica de la sociedad, llevando a que el reconocimiento dependa de la valoración de la contribución a los objetivos que la sociedad considere como valiosos (Thayer, 2013).

\section{La interseccionalidad en la experiencia de las mujeres migrantes latinoamericanas en empleos masculinizados}

La dificultad de tratar el género separado de otras categorías de opresión se ha convertido en fundamental en el abordaje interseccional de los estudios feministas (Troncoso, Galaz y Álvarez, 2017). La mirada articulada nos permite aproximarnos a las diferencias que posibilitan o niegan el reconocimiento de las migrantes, considerando que no puede comprenderse ni "mujer" ni "migrante" como categorías universales que agrupan a todas las mujeres y a todos/as los/as migrantes, y que incluso las "mujeres migrantes" representen un grupo homogéneo con las mismas características y experiencias. En estudios de migración (Stefoni y Stang, 2017) se ha sugerido la importancia de incorporar la perspectiva interseccional, que posibilita la comprensión del vínculo y la relación entre varias categorías de desigualdad, como "raza", nacionalidad, clase y género, las que moldean las experiencias migratorias.

El concepto de interseccionalidad fue acuñado en 1989 por Crenshaw (Cho, Crenshaw y McCall, 2013; Troncoso et al., 2019; Viveros, 2016;), buscando estudiar identidades intersectadas en escenarios de opresión o discriminación. Retomando el legado de las ponentes clásicas de la interseccionalidad, el feminismo decolonial latinoamericano continúa su teorización, sin embargo, acercándose más a los planteamientos de Collins (Cubillos, 2015), al hacer una reinterpretación en clave crítica que incluye, además del androcentrismo y la misoginia, el carácter racista y eurocéntrico (Espinoza, 2016). Ha sido descrito como "heredero directo del feminismo negro, de color y tercermundista en los EE.UU., en su crítica a la teorización feminista clásica centrada en género y su propuesta de un tratamiento interseccional de las opresiones (de clase, raza, género y sexualidad)" (Espinoza, 2016, p. 151).

Sobre el concepto de interseccionalidad, citando a Galaz et al. (2019), Collins y Bilge (2016) lo mencionan como una forma de comprender y analizar el mundo, las personas 
y sus experiencias, allí donde se requiere la comprensión de varios factores que se influyen mutuamente a fin de explicar distintos acontecimientos en la vida de las personas, pues estos serían difícilmente entendibles como producto de un único factor. Plantean también que brinda una forma de análisis de procesos generados por la interacción de distintos factores que pueden afectar las oportunidades de desarrollo y ejercicio de derechos por el hecho de que los sujetos se encuentren en esa intersección de desigualdades (Galaz et al., 2019). Para Curiel (2014), el concepto implica un reconocimiento de la diferencia colonial desde categorías intersectadas presentadas como ejes de subordinación, que fueron separadas y autónomas, pero que luego se cruzaron. Sin embargo, considera que la interseccionalidad no indaga lo suficiente en la producción de las diferencias en las experiencias de muchas mujeres, tendiendo, por tanto, a "un multiculturalismo liberal que pretende reconocer las diferencias, incluyéndolas en un modelo diverso, pero que no cuestiona las razones que provocan la necesidad de esa inclusión. En otras palabras, sigue definida desde el paradigma moderno occidental eurocéntrico" (Curiel, 2014, p. 55).

En este sentido, Lugones (2005) se refiere al desplazamiento de una lógica de la opresión que ha posibilitado que las dominaciones culturales y de género se hayan enmascarado como multiculturales o feministas hacia una lógica de la resistencia.

La máscara feminista es la que se opone a una versión de la femineidad que se ha atribuido únicamente a mujeres que, en términos de raza, clase y sexualidad, se han entendido como subordinadas sólo a los burgueses blancos. Este feminismo ha sido complaciente con la sumisión de todas las demás mujeres. (Lugones, 2005, pp. 61-62).

La subordinación de las mujeres frente al poder de hombres blancos se intenta extrapolar a la subordinación de todas las mujeres (Lugones, 2005), dejando por fuera a muchas que viven la intersección de categorías de opresión. En consecuencia, las situaciones y experiencias de mujeres migrantes latinoamericanas que trabajan en empleos masculinizados se abordan considerando las distintas categorías de opresión interrelacionadas, evitando caer en tratar el fenómeno desde las formas dominantes de comprensión de este.

\section{Metodología}

El estudio base de este artículo fue cualitativo, de carácter exploratorio-descriptivo (Monje, 2011). Se propuso abordar la subjetividad de las migrantes que se desempeñan en trabajos masculinizados, entendiendo desde allí cómo experimentan las relaciones e interacciones y cómo perciben la justicia de género ligada al reconocimiento. Con este objetivo, se realizaron entrevistas en profundidad a ocho mujeres migrantes latinoamericanas (de Colombia y Venezuela) pertenecientes al grupo poblacional económicamente activo (Organización Internacional para las Migraciones [OIM], 2018), hispanohablantes y sin doble nacionalidad (en relación con la chilena), que laboraran en un empleo masculinizado y que hubieran permanecido en él entre seis meses y cuatro años. Se consideraron trabajos masculinizados aquellos que fueran 
predominantemente realizados por hombres (Díaz, 2014), sobre la base de una búsqueda empírica y corroborando esta información desde la percepción de las entrevistadas, quienes en todos los casos confirmaron dicha prevalencia, siendo incluso su participación pionera en ese puesto de trabajo. Se accedió por medio de la técnica bola de nieve a entrevistadas de Colombia y Venezuela, en consideración a que son dos de las nacionalidades hispanohablantes con mayor presencia en Chile (junto con la peruana), representando un $31 \%$ y $12 \%$ de la población femenina migrante en la actualidad, respectivamente (INE y DEM, 2020). La Tabla $\mathrm{N}^{\circ} 1$ entrega las principales características de la muestra:

Tabla $\mathbf{N}^{\circ}$ 1: Características de las entrevistadas

\begin{tabular}{|c|c|c|c|c|c|c|}
\hline da & dad & dad & $\begin{array}{l}\text { Profesión } \\
\text { de origen }\end{array}$ & $\begin{array}{l}\text { Empleo } \\
\text { actual }\end{array}$ & $\begin{array}{c}\text { Tipo de } \\
\text { trabajo }\end{array}$ & $\begin{array}{c}\text { Tiempo } \\
\text { de } \\
\text { permanencia } \\
\text { en Chile }\end{array}$ \\
\hline 1 & $\begin{array}{l}\text { Colombi } \\
\text { ana }\end{array}$ & $\begin{array}{c}38 \\
\text { años }\end{array}$ & $\begin{array}{r}\text { Curso de } \\
\text { inyectología }\end{array}$ & Conserje & $\begin{array}{c}\text { No } \\
\text { calificado }\end{array}$ & $\begin{array}{l}1 \text { año y } \\
6 \text { meses }\end{array}$ \\
\hline 2 & $\begin{array}{l}\text { Colombi } \\
\text { ana }\end{array}$ & $\begin{array}{l}41 \\
\text { años }\end{array}$ & $\begin{array}{l}\text { Auxiliar en } \\
\text { enfermería }\end{array}$ & Conserje & $\begin{array}{c}\text { No } \\
\text { calificado }\end{array}$ & 3 años \\
\hline 3 & $\begin{array}{l}\text { Venezola } \\
\text { na }\end{array}$ & $\begin{array}{l}25 \\
\text { años }\end{array}$ & $\begin{array}{l}\text { Ingeniera } \\
\text { en } \\
\text { telecomunica- } \\
\text { ciones no } \\
\text { graduada }\end{array}$ & $\begin{array}{l}\text { Programa } \\
\text { dora }\end{array}$ & $\begin{array}{l}\text { Calificad } \\
\quad \mathrm{O}\end{array}$ & 3 años \\
\hline 4 & $\begin{array}{l}\text { Venezola } \\
\text { na }\end{array}$ & $\begin{array}{c}26 \\
\text { años }\end{array}$ & $\begin{array}{l}\text { Ingeniera } \\
\text { eléctrica }\end{array}$ & Ingeniera & $\begin{array}{l}\text { Calificad } \\
\text { o }\end{array}$ & $\begin{array}{l}1 \text { año y } \\
7 \text { meses }\end{array}$ \\
\hline 5 & $\begin{array}{l}\text { Venezola } \\
\text { na }\end{array}$ & $\begin{array}{r}24 \\
\text { años }\end{array}$ & $\begin{array}{l}\text { Ingeniera } \\
\text { electrónica }\end{array}$ & Ingeniera & $\begin{array}{l}\text { Calificad } \\
\text { o }\end{array}$ & $\begin{array}{r}1 \text { año y } \\
\text { seis meses }\end{array}$ \\
\hline 6 & $\begin{array}{l}\text { Venezola } \\
\text { na }\end{array}$ & $\begin{array}{l}26 \\
\text { años }\end{array}$ & $\begin{array}{l}\text { Ingeniera } \\
\text { electrónica }\end{array}$ & Ingeniera & $\begin{array}{l}\text { Calificad } \\
\quad \text { o }\end{array}$ & 8 meses \\
\hline 7 & $\begin{array}{l}\text { Venezola } \\
\text { na }\end{array}$ & $\begin{array}{l}32 \\
\text { años }\end{array}$ & $\begin{array}{l}\text { Comunicac } \\
\text { ión social y } \\
\text { periodismo }\end{array}$ & $\begin{array}{l}\text { Bombera } \\
\text { de bencina }\end{array}$ & $\begin{array}{c}\text { No } \\
\text { calificado }\end{array}$ & 4 años \\
\hline 8 & $\begin{array}{l}\text { Venezola } \\
\text { na }\end{array}$ & $\begin{array}{l}56 \\
\text { años }\end{array}$ & $\begin{array}{l}\text { Pedagogía, } \\
\text { docencia } \\
\text { universitaria }\end{array}$ & $\begin{array}{l}\text { Encargad } \\
\text { a de } \\
\text { seguridad y } \\
\text { anfitriona de } \\
\text { estacio- } \\
\text { namiento }\end{array}$ & $\begin{array}{c}\text { No } \\
\text { calificado }\end{array}$ & $\begin{array}{r}1 \text { año y } \\
\text { seis meses }\end{array}$ \\
\hline
\end{tabular}

Fuente: elaboración propia a partir de datos obtenidos de las participantes.

Las entrevistas fueron grabadas y transcritas buscando garantizar el rigor científico. Se firmó un consentimiento informado mediante el cual se indicó a las participantes su carácter voluntario, el derecho a retirarse en el momento que quisieran, el resguardo de su identidad y que las entrevistas serían utilizadas únicamente con fines académicos, de manera de cumplir con los requisitos éticos de la investigación y como una muestra de 
respeto hacia las participantes. ${ }^{1}$ Además, la entrevistadora acordó con las participantes lugares y horarios favorables y cómodos para ellas, poniéndose al servicio de garantizar la confianza y el bienestar necesario.

El análisis de los datos se realizó usando la técnica de análisis de contenido, que considera las siguientes categorías: compensación económica, estima social y autovaloración, ligadas al reconocimiento en su esfera del principio del éxito (Honneth, 2006). El proceso incluyó el establecimiento de subcategorías y códigos, que emergieron del corpus empírico al clasificar elementos de las entrevistas y al codificar los relatos a partir de las tres categorías vinculadas con el reconocimiento, analizadas desde la interseccionalidad. La codificación se hizo en una matriz Excel, ubicando cada fragmento de la entrevista bajo una categoría y subcategoría, y asignándole un código (ver tablas $\mathrm{N}^{\circ} 2,3$ y 4). Posteriormente, se redactaron los resultados, evidenciando el discurso literal o referenciado de las entrevistadas, garantizando la rigurosidad científica del análisis. Se abordó en el análisis conjuntamente trabajos calificados y no calificados porque se quiso evidenciar cómo iban asemejándose o diferenciándose las experiencias de las migrantes en trabajos que cumplían con una u otra característica.

Tabla $\mathbf{N}^{\circ}$ 2: Compensación económica

\begin{tabular}{ccccc}
\hline Categoría & \multicolumn{4}{c}{$\begin{array}{c}\text { Compensación } \\
\text { económica }\end{array}$} \\
\hline $\begin{array}{c}\text { Subcategoría } \\
\text { S }\end{array}$ & Salario & Beneficios & $\begin{array}{c}\text { Necesidade } \\
\text { s }\end{array}$ & Situación física \\
\hline Código & Ajuste salarial & $\begin{array}{c}\text { Estabilidad } \\
\text { laboral }\end{array}$ & $\begin{array}{c}\text { Necesidad } \\
\text { económica }\end{array}$ & Cansancio \\
\cline { 2 - 5 } & $\begin{array}{c}\text { Desigualdad } \\
\text { de salarios }\end{array}$ & $\begin{array}{c}\text { Desigualda } \\
\text { d de } \\
\text { Beneficios }\end{array}$ & $\begin{array}{c}\text { Regulación } \\
\text { migratoria }\end{array}$ & $\begin{array}{c}\text { Malestares de } \\
\text { salud }\end{array}$ \\
\cline { 2 - 5 } & Salario justo & $\begin{array}{c}\text { Entrega de } \\
\text { Beneficios }\end{array}$ & \\
\hline
\end{tabular}

Fuente: elaboración propia a partir de datos obtenidos de las entrevistas.

\footnotetext{
${ }^{1}$ El proyecto de investigación Fondecyt 1180590, en el que se inserta el estudio base de este artículo, contó con la aprobación del comité de ética institucional.
} 
Tabla $\mathbf{N}^{\circ} 3$ Estima social

\begin{tabular}{|c|c|c|c|c|}
\hline \multicolumn{5}{|c|}{ Estima social } \\
\hline $\begin{array}{l}\text { Subcategorí } \\
\text { as }\end{array}$ & $\begin{array}{l}\text { Relación } \\
\text { con } \\
\text { el o la } \\
\text { jefe/a }\end{array}$ & $\begin{array}{c}\text { Relación con } \\
\text { compañeros y } \\
\text { compañeras de trabajo }\end{array}$ & $\begin{array}{l}\text { Relación } \\
\text { con } \\
\text { clientes }\end{array}$ & $\begin{array}{l}\text { Reacción de } \\
\text { familiares } \\
\text { y amigos/as }\end{array}$ \\
\hline \multirow[t]{11}{*}{ Código } & Valoración & Amabilidad & $\begin{array}{l}\text { Amabilid } \\
\text { ad }\end{array}$ & Apoyo \\
\hline & Buen trato & Rechazo & $\begin{array}{l}\text { Falta de } \\
\text { respeto }\end{array}$ & Orgullo \\
\hline & $\begin{array}{l}\text { Imparcialid } \\
\text { ad }\end{array}$ & Xenofobia & & Crítica \\
\hline & & Sexismo & & $\begin{array}{c}\text { Feminizació } \\
\mathrm{n} \text { del }\end{array}$ \\
\hline & & Humillación & & $\begin{array}{c}\text { trabajo } \\
\text { masculinizado }\end{array}$ \\
\hline & & Envidia & & \\
\hline & & Traición & & \\
\hline & & Traspaso de tareas & & \\
\hline & & $\begin{array}{l}\text { Herramientas de } \\
\text { Autoprotección }\end{array}$ & & \\
\hline & & Resiliencia & & \\
\hline & & Valoración & & \\
\hline
\end{tabular}

Fuente: elaboración propia a partir de datos obtenidos de las entrevistas.

Tabla $N^{\circ}$ 4: Autovaloración

\begin{tabular}{|c|c|c|c|c|}
\hline Categoría & & Autovaloración & & \\
\hline Subcategorías & Aportes & $\begin{array}{c}\text { Comparación con } \\
\text { los/as } \\
\text { compañeros/as }\end{array}$ & $\begin{array}{c}\text { Ejecución } \\
\text { del } \\
\text { trabajo }\end{array}$ & Autopercepción \\
\hline \multirow[t]{3}{*}{ Código } & $\begin{array}{l}\text { Ligados a la } \\
\text { nacionalidad }\end{array}$ & Más valor & $\begin{array}{c}\text { Amor por el } \\
\text { trabajo }\end{array}$ & $\begin{array}{c}\text { Autoconfianz } \\
a\end{array}$ \\
\hline & $\begin{array}{l}\text { Ligados a } \\
\text { roles } \\
\text { femeninos }\end{array}$ & Menos valor & $\begin{array}{l}\text { Excelencia en } \\
\text { el desempeño }\end{array}$ & Inseguridad \\
\hline & $\begin{array}{c}\text { Ligados a } \\
\text { estereotipos } \\
\text { femeninos }\end{array}$ & $\begin{array}{l}\text { Complementaried } \\
\text { ad }\end{array}$ & & \\
\hline
\end{tabular}




\section{Resultados y análisis}

De las mujeres entrevistadas, las dos trabajadoras colombianas llegaron a Chile en busca de mejores oportunidades económicas, teniendo claro desde antes de su arribo que su intención era buscar un trabajo que no necesitara mayor calificación, pues sabían, por voz de conocidos que habían llegado previamente a Chile, que en el país había buenas posibilidades para migrantes que quisieran hacer trabajos que ciudadanos/as chilenos/as no querían desarrollar y que además lo hicieran por menor salario (Thayer, 2012). Por otra parte, las mujeres venezolanas migraron debido a la situación política y económica de su país de origen; las seis iniciaron sus estudios universitarios y solo una de ellas no alcanzó a graduarse porque tuvo que salir del país intempestivamente, sin embargo, complementó sus estudios de programación en Chile; las otras cinco son profesionales, una inclusive tiene un posgrado. Las tres ingenieras y la programadora laboran en empleos concordantes con sus estudios, aunque lo hacen en áreas que no habían explorado en su país de origen y que se distancian de sus experiencias e intereses originales. Las otras dos mujeres venezolanas, aunque profesionales, no han podido encontrar en Chile un empleo ligado directamente a su profesión, por lo que han recurrido a trabajar en empleos no calificados.

\section{Compensación económica que reciben las migrantes que laboran en empleos masculinizados}

Como una reafirmación de la brecha salarial existente entre mujeres y hombres en el mundo laboral, las migrantes no encuentran satisfacción con el salario recibido, calificándolo incluso de "miserable", haciendo evidente así la pretensión de un aumento de este. Argumentan la existencia de diferencias entre el monto que ellas reciben y el de sus compañeros hombres chilenos y extranjeros; sin embargo, racionalizan esta diferencia basada en un asunto de experiencia y no como una desigualdad explícita de género, pareciéndoles invisible ese factor. A pesar de no estar a gusto con el salario actual, quienes se desempeñan en empleos calificados perciben como ganancia adicional al salario la obtención de "experiencia", variable que más adelante les permitirá mejorar sus currículums y proyectar un cambio de empleo en el que tengan una mejor retribución salarial.

Por otra parte, estas trabajadoras migrantes confunden los beneficios no económicos con el cumplimiento de las obligaciones legales que debe garantizar todo empleador, a pesar de que en sus países de origen también son asuntos obligatorios. Nos referimos al pago de la salud (FONASA), el pago de la Administradoras de Fondos de Pensionales (AFP), la colación, el uniforme, entre otras, disociándose de esta forma la percepción de que se trata de derechos laborales. Solo una de las entrevistadas manifestó recibir un beneficio relativo a salud y esparcimiento al mismo tiempo, como el acceso al gimnasio. Se toma también en consideración como beneficio el hecho de tener un contrato indefinido como garantía de estabilidad. Esto se vincula directamente con la realidad migratoria, respecto 
de la cual muchas trabajadoras, a pesar de tener su estatus migratorio reconocido legalmente, en ocasiones laboran informalmente, sin contrato de trabajo, lo que es un indicador de precariedad laboral (Leiva et al., 2017).

En algunos casos, el empleador entrega bonos o aguinaldos, sin embargo, el valor que reciben es inferior al de sus compañeros hombres. Algunas trabajadoras argumentan que estas situaciones se darían por una cuestión de rendimiento, aunque no hay criterios explícitos para medir este aspecto; y en otras, perciben que no existen razones aparentes, lo que evidenciaría una desigualdad arbitraria por razones de género, que en este caso sí interpretan como discriminación.

Aunque se evidencia que no hay una satisfacción y agrado absoluto por los actuales empleos, y los sueldos que reciben no se ajustan a sus expectativas, permanecen en ellos por necesidad económica, puesto que son la mejor opción del momento y porque les permite cumplir con los requisitos indispensables para acceder a una visa definitiva, tales como tener un número mínimo de cotizaciones previsionales. Para estas migrantes, las razones antes expuestas justifican el trabajar en empleos no calificados aceptando desempeñarse en condiciones de inseguridad o peligro, como jornadas de trabajo nocturno, salir de turno en la madrugada y desplazarse por lugares públicos inseguros, permanecer solas en sus puestos de trabajo (bencinera de estación de servicios en turno de noche) o cumplir la función de ser responsables de la seguridad de un edificio sin contar con la preparación para repeler asaltos o robos, entre otras.

Gracias a esta empresa pude tomar las cotizaciones para la visa definitiva, o sea eso era vital. Si no hubiese sido por eso, me hubiese ido antes en verdad. (Entrevistada 7, bombera de bencina, 32 años).

Tanto para mujeres con empleos calificados como no calificados, su principal preocupación es mantener el empleo actual, pues es su ingreso permanente, lo que las lleva a evitar cualquier contingencia $y$, por ende, a aceptar incomodidades o incumplimientos laborales que pongan en riesgo la pérdida de su fuente actual de ingresos, fragilizándose a futuro la posibilidad de acceder a un empleo mejor. Durante el 2019, debido al colapso de las atenciones a migrantes en el Departamento de Extranjería y Migración, la Policía de Investigaciones y el Registro Civil -que impedía realizar renovaciones expeditas del carnet de identidad- manifestaron que era la segunda razón más poderosa para que estas prefiriesen mantenerse en el empleo actual, aunque no les conformara tanto en el plano del reconocimiento salarial como de la valoración social. Esto evidencia una falta de reconocimiento por parte del Estado, lo que responde a las barreras que este impone a este grupo poblacional en desmedro de las garantías laborales de las migrantes.

Las entrevistadas manifestaron que, previo a sus empleos actuales, pasaron por otros, algunos feminizados y otros de carácter mixto. Todas cuentan con trayectorias y relatos de tratos denigrantes y de maltrato verbal por parte de sus empleadores, situaciones que señalan debieron "aguantar" por la necesidad de mantener sus empleos y fuentes ingresos en pos de no romper el proceso de regularización migratoria que buscaban 
conseguir. Estas mujeres verbalizan que hay una conciencia del aprovechamiento de los empleadores ante su situación migratoria, explicitando abusos de poder y xenofobia.

Hasta este punto las migrantes parecieran tener una experiencia más o menos parecida frente a su participación en un trabajo masculinizado, en los que reciben remuneraciones inferiores a lo esperado y se enfrentan a situaciones de malos tratos y precariedad laboral. Al mismo tiempo visibilizan como "beneficios" prestaciones que constituyen obligaciones legales, demostrando con ello, como se ha indicado en estudios previos, que las migrantes son parte de un conglomerado dentro de la fuerza laboral que accede a empleos con menos ingresos y mayor precariedad laboral (Acosta, 2013), y que ello se podría relacionar con una baja concepción de ser sujetas de derechos laborales. Así y todo, las experiencias de mujeres migrantes en empleos masculinizados en Santiago de Chile no pueden encasillarse como únicas, semejantes y uniformes entre sí, sino que se presentan diferencias cuando se observa la intersección de género, nacionalidad y tipo de trabajo que desempeñan (calificado o no calificado).

De tal forma, un aspecto recurrente en mujeres migrantes que realizan trabajos no calificados masculinizados fue la fatiga física a la que se ven expuestas, debido a su exposición a largas jornadas de trabajo que en ocasiones pueden dudar más de 12 horas; al pluriempleo, ya que la mayoría tiene trabajos complementarios secundarios al principal, que amplía sus jornadas laborales diarias y semanales. Es así como algunas trabajan alrededor de ocho horas (a veces más) en su trabajo "formal" principal, al que se le agrega la realización de una segunda e incluso tercera actividad remunerada, como hacer aseo en casas u oficinas; y a la doble jornada, debido a que son las principales responsables de las labores domésticas de su hogar. La sobrecarga laboral las lleva a disminuir sus horas de sueño, llegando en algunos casos a ser solo de un par de horas, para salir al otro día a repetir la misma rutina sobreexigida. En consecuencia, soportan agotamiento y estrés físico y mental por razones económicas. Esto da cuenta de un panorama existencial dificultoso, que es resultado tanto de las condiciones de realización del trabajo reproductivo como productivo de las mujeres migrantes que realizan empleos no calificados, lo que denota una precariedad objetiva y subjetiva (Piñeiro, 2011). Entiéndase objetiva a la luz de los bajos salarios, horarios extensos y falta de estabilidad; y subjetiva, manifestada en agotamiento, estrés e incertidumbre frente a la inestabilidad laboral.

Sumado a esto, algunos jefes permiten que las trabajadoras no tomen el tiempo de vacaciones, sino que lo trancen por más trabajo remunerado, cometiendo de este modo una falta legal, pues en el caso del descanso anual, se trata de un derecho irrenunciable. Evidentemente, todo esto acarrea consecuencias en su salud integral.

\section{Estima social, la valoración por parte de los/as otros/as}

En la cotidianidad laboral de las mujeres migrantes se revelan las relaciones con los/as jefes/as, los/as compañeros/as de trabajo chilenos/as o de otras nacionalidades y con 
los/as clientes/as. Por otra parte, el vínculo familiar se evidencia como un aspecto clave que se resalta en sus relatos en cuanto a la valoración que parientes y personas cercanas hacen respecto a su participación en un empleo masculinizado.

En general, las mujeres que se desempeñan en empleos calificados perciben que poseen buenas relaciones con jefes/as directos/as aparentemente imparciales en cuanto a género y nacionalidad (en comparación con el trato que reciben ellas y sus compañeras chilenas o compañeros hombres chilenos o migrantes), ambientes laborales donde observan existe valoración. Sin embargo, quienes tienen empleos no calificados destacan experiencias en las que han sido descalificadas por las jefaturas, quienes se respaldan y argumentan en "falsas justificaciones culturales" sobre la conducta propia de los empleadores en el país.

Bueno, sí, por ejemplo, yo le comenté a mi jefa la posibilidad de estudiar portugués, dos horas a la semana, solo dos horas a la semana, y como ella hacía el horario semanalmente, pues no le costaba nada acomodarlo, y según ella no me lo daba porque tenía que ser igual para todo el mundo, pero no todo el mundo le estaba pidiendo oportunidad de estudiar y yo sí quería, quería complementar un poco y lo iba a pagar con mi plata, entonces, sí me pareció muy injusto (...) No, es un tema de cultura, porque ella me decía que aquí en Chile no se daba, que era muy difícil que las empresas te dieran oportunidad de estudiar porque el empleador no quiere que tú progreses. (Entrevistada 7, bombera de bencina, 32 años).

En cuanto a la relación laboral con pares en el lugar de trabajo, surgen dos tipos de experiencias ligadas directamente con el hecho de tener o no una profesión de base, independientemente del tipo de trabajo que se desarrolle (calificado o no calificado), vinculando la valoración de los pares respecto de su desempeño con una cuestión de posición o clase social. Para quienes cuentan con una profesión de base, se evidencian entornos laborales culturales favorables y amables, caracterizados por el compañerismo y respeto; sin embargo, en los casos en que no hay una profesión de base, se presentan experiencias de maltrato, xenofobia, sexismo, racismo, entre otras formas de discriminación, lo que lleva estas mujeres migrantes a generar estrategias de resistencia para lograr sobrellevar las situaciones descritas con el fin de garantizar su permanencia en los empleos y así alcanzar el objetivo económico o migratorio propuesto.

De tal forma, las diferencias de nacionalidad, género y tipo de empleo se intersectan con las diferencias de clase, que se manifiestan en que las migrantes que trabajan en empleos masculinizados no calificados y que no tienen profesión de base se enfrentan a una discriminación que incluye estos ejes de diferenciación. Tanto las categorías de diferenciación como el pensamiento categorial actúan como instrumentos de opresión, siendo la multiplicidad ignorada a partir de las categorías que no involucran a quienes viven la intersección de condiciones de opresión (Lugones, 2005). Es allí donde las migrantes que trabajan en empleos no calificados masculinizados podrían no ser visibilizadas porque no son evidentes las categorías que se cruzan, especialmente la de género. Pero al observar la intersección entre género, nacionalidad y calificación (clase) se vislumbra la opresión y la falta de reconocimiento que reciben. 
Cuando yo llegué, mis compañeros me discriminaron, que porque aquí en este puesto nunca había habido mujeres de conserje, y que la única mujer era la señora del aseo, y que ya venían los extranjeros a invadir el espacio porque eran todos los conserjes aquí antes chilenos. (Entrevistada 1, conserje, 38 años).

El trato con clientes/as es común en ciertos oficios y profesiones, pero la experiencia que se genera en esta relación no resulta muy favorable entre las entrevistadas. Malos momentos generados por una discriminación basada en cuestión de sexo, el tipo de trabajo y clase han sido constantes, mencionando su ocurrencia en mayor proporción que la que reciben por parte de empleadores/as o colegas. A pesar de ello, se destacan algunos clientes con los que han tenido una relación cordial y de amabilidad, y que incluso han recibido comentarios directos en los que estos les reconocen cualidades como el buen servicio. Lo anterior es autopercibido por las entrevistadas como una característica común en la cultura de trato en sus países de origen, lo que se vincula con lo previamente mencionado respecto a las representaciones positivas que ellas hacen de sí mismas, elaboradas a partir de una asociación entre la nacionalidad y el trabajo (Trpin, 2007).

Familiares y amigos/as expresan opiniones sobre la valoración que hacen con respecto a la presencia de las mujeres migrantes en empleos masculinizados. Según testimonios de las entrevistadas, se evidencia y reconoce un respaldo y orgullo familiar hacia quienes desempeñan empleos calificados; sin embargo, ese respaldo carece de orgullo cuando las mujeres trabajan en empleos no calificados, pero tienen profesión de base, aun cuando manifiestan que es sabido, tanto por ellas como por sus familiares, que no es el empleo "elegido", sino uno que responde a una necesidad puntual, de carácter temporal. Por último, las mujeres que no cuentan con una profesión de base y que además tienen un empleo no calificado, reciben por parte de sus familiares comentarios en los que expresan el temor que les causa que ellas realicen un trabajo que denominan como "de hombre", asociando así la labor que desempeñan al apartamiento de los roles de género tradicionales.

La referencia a estereotipos de género es constantemente verbalizada por los/as amigos/as cercanos/as, quienes desde su ideal tradicional buscan resaltar los aportes y atributos femeninos en la labor que realizan las mujeres en un empleo masculinizado. Asimismo, manifiestan preferir que ellas se desempeñen en labores que constituyen una extensión de los roles tradicionales de género, como el hacer aseo en una bencinera o manejar la caja de un estacionamiento, expresando así un imaginario que no reconoce la capacidad de las mujeres para ejecutar tareas que hegemónicamente han sido asociadas a los hombres, o que les resulta forzada y poco "natural". De tal forma, se encasilla a las mujeres como subordinadas e incapaces, negando el reconocimiento en cuanto a su aporte dentro de la esfera del éxito (Honneth, 2006) y reproduciendo diferenciaciones en las que el reconocimiento de un grupo está dado por las condiciones de vida de otros (Lugones, 2005). Se observa, entonces, que las mujeres en empleos calificados asociados a hombres carecen del pleno reconocimiento de sus familiares cercanos porque Se observa, entonces, que las mujeres en empleos calificados asociados a hombres carecen del pleno reconocimiento de sus familiares cercanos, porque se sigue 
sobrevalorando culturalmente a los hombres que ejercen dichos empleos profesionales más que a las mujeres. Paradójicamente, si este se diera en una esfera feminizada sería considerado como extensión del rol de género y por tanto igualmente infravalorado.

\section{Relatos sobre la autovaloración}

El aporte que las mujeres migrantes en empleos masculinizados perciben que realizan en su trabajo está directamente ligado a su autovaloración. De acuerdo con su percepción, aspectos culturales de buen trato y amabilidad, ligados a sus nacionalidades de origen, como la puntualidad, la responsabilidad y la amabilidad de trato en el trabajo, son reconocidos como aportes importantes que ellas hacen en sus trabajos, pues aseguran que estos no existen en Chile en la misma magnitud en que se dan en sus países. Al ser resaltados estos aspectos por ellas, pueden ser interpretados como mecanismos de resistencia (Galaz, et al., 2019) frente a la discriminación por xenofobia que algunas reconocen haber experimentado.

En este sentido, su condición de trabajadoras colombianas o venezolanas es autopercibida de forma positiva, valoración elaborada al compararse con otros/as trabajadores/as nativos/as de Chile. Esto evidencia una asociación entre las representaciones nacionales y laborales que las mujeres migrantes tienen de sí mismas en contraposición a connotaciones peyorativas sobre su nacionalidad, como en el caso presentado por Trpin (2007) sobre hijos de migrantes chilenos en Argentina, donde en lugares cotidianos los argentinos y chilenos reproducen la categoría "chileno" como recurso de inserción laboral, lo que implica no solo conocer el trabajo sino reproducir ciertos atributos homogéneos de la chilenidad. En el caso concreto presentado por Trpin (2007):

La representación de que el chileno es un "buen trabajador" que nunca "pide de más" es una construcción que se erige en oposición a los trabajadores temporarios que llegaban del norte argentino. La chilenidad garantiza la disciplina de trabajo como un atributo a destacar frente al empleador, como una manera de presentarse en oposición a la connotación peyorativa que a veces transmite "ser chileno" en la Patagonia. (p. 247).

En este sentido, las características positivas que las migrantes destacan sobre sí mismas podrían surgir como un mecanismo de resistencia que, como describen Galaz et al. (2019), puede ser tanto abiertamente confrontacional, o bien, expresión de pequeñas resistencias cotidianas.

Por otra parte, se mencionan aportes ligados a características y tareas hegemónicamente consideradas como femeninas que realizan de manera complementaria a su función laboral principal, no presente de manera explícita en la descripción del cargo, como asear el baño de la conserjería o encargarse de los detalles y la estética de una aplicación web. Estos son algunos de los aportes por los que perciben destacarse en un empleo 
masculinizado, ligando constantemente dichas contribuciones adicionales a lo que se espera de ellas en tanto mujeres, según una mirada estereotipada de género.

Bueno, en este caso, como nosotros hacemos mucho la parte de programación y desarrollo, desarrollamos también aplicaciones móviles, aplicaciones web y todo eso y, obviamente, lo primero que hace una mujer es el toque de los detalles: esto por aquí, esto por allá, un color, ¿sabes? Hay detalles que, aunque el programa funcione perfectamente, una como mujer lo ve y dice: "Esto puede mejorar; veo esto y si lo veo así no me convence, colócalo de otra manera", entonces, esto ha sido como uno de los aportes, no se sí el más significativo, pero que ha sido como más notorio. (Entrevistada 5, ingeniera electrónica, 24 años).

Se tiene la idea de hacer las cosas bien en el trabajo, a pesar de no estar a gusto con la función o con el salario, así como se evidencia empeño y dedicación a la tarea en todos los relatos de las entrevistadas. Sin embargo, y como una cuestión muy ligada a estereotipos de género, se manifiesta que, aunque expresan amor por el trabajo, señalan que no pueden amarlo en la misma medida en que lo hacen los hombres. Lo anterior da cuenta del vínculo que se crea entre el hecho de tener más o menos "amor" hacia el trabajo y un asunto de género, respecto del cual consideran que los hombres se enlazan identitariamente al trabajo productivo con una predominancia mayor que las mujeres, cuya presencia en el trabajo correspondería a una oportunidad en la que es normal estar en una posición de subordinación, basada en que la relación entre sexo y trabajo productivo se vincula con la posición de género culturalmente respaldada.

No obstante lo anterior, hay consenso en sus relatos en torno a que ninguna se autopercibe de manera explícita con menor valoración por ser mujer ni por ser migrante. Además consideran que como el proceso migratorio es tan complejo y han tenido que pasar por tantas dificultades en ese sentido, que están seguras de poder lograr cualquier desafío laboral que se propongan. Su autopercepción positiva se confronta con la menor valoración que indican percibir por parte de clientes/as y colegas, y en menor proporción por parte de jefaturas en los casos en que no cuentan con una profesión de base.

En este sentido se presenta una paradoja clave, que es que quienes tienen empleos calificados reciben mayor valoración por parte de familiares, amigos/as, jefes y pares, pero construyen una imagen de sí mismas cuyos aportes en un trabajo masculinizado adquieren menor relevancia que los de sus compañeros hombres (independientemente de su nacionalidad). Por el contrario, quienes se desempeñan en empleos no calificados, significan sus labores al contrario, pues a pesar de recibir críticas y de ser constantemente discriminadas, su autoimagen es fuerte y perciben dar un aporte más importante que el de los demás. Esto podría ser interpretado como un mecanismo de resistencia -autopercibirse fuertes y autovalorar su aporte- frente al cruce de opresiones al que se enfrentan -sexo, nacionalidad y tipo de empleo- y al hecho de se les niega el reconocimiento desde múltiples ejes. 


\section{Conclusiones}

En este artículo, que buscó comprender la forma en que las migrantes que trabajan en empleos masculinizados en Santiago de Chile experimentan las relaciones e interacciones sociales - a partir de su percepción de justicia de género basada en el reconocimiento en sus entornos laborales-, se evidencia que la injusticia que experimentan estas mujeres depende de la valoración cultural de las capacidades de éxito (Honneth, 2006). En este caso, dadas dentro de los entornos laborales masculinizados. Así, la falta de reconocimiento que perciben recibir se genera principalmente por razones de estereotipos de género, en tanto mujeres que realizan una ocupación masculinizada y, por tanto, cultural y socialmente valorada por ser "trabajo de hombres", factor que influye paradojal y positivamente en su autovaloración, desde la que construyen una imagen fuerte de sí mismas, pero que se relaciona de manera conflictiva con los imaginarios sociales tradicionales de género.

Por otra parte, se observa una articulación entre el concepto de reconocimiento e interseccionalidad para comprender las distintas formas de injusticia y falta de reconocimiento que afectan a las migrantes que trabajan en empleos masculinizados, considerando distintas características de diferenciación, tales como género y nacionalidad, además del tipo de trabajo que realizan (calificado o no calificado), siendo un elemento leído en clave de clase social.

Las migrantes que trabajan en empleos masculinizados exhiben trayectorias de vida diferenciadas con respecto a sus pares varones y pares chilenos/as. Sin embargo, no puede establecerse una unificación u homogenización sobre la opresión que ellas viven ni sobre la falta de reconocimiento a la que se enfrentan. Este artículo clarifica que no puede abordarse su nacionalidad o condición de migrante sin observar otros ejes de diferenciación, así como no puede considerarse únicamente desde el punto de vista de género. Se evidencia en las relaciones laborales que se generan una falta de reconocimiento hacia las migrantes en trabajos masculinizados, la que se acrecienta al intersectarse con otras categorías de diferenciación, tales como clase social, tipo de trabajo y, en ocasiones, el color de piel. Al mismo tiempo queda claro que entre más categorías se intersectan, más herramientas de resistencia se generan para hacer posible su permanencia en el empleo y enfrentar la discriminación, resaltando sus características positivas asociadas a su nacionalidad o género, las que contribuyen a acrecentar una autovaloración favorable.

Se concluye que existen injusticias de carácter distributivo, en cuanto a las compensaciones económicas. Estas inequidades evidencian que los trabajos masculinizados están marcados por atributos que la sociedad ha asignado a los hombres, reproduciendo prejuicios acerca de los límites de las capacidades de las mujeres para desarrollar esas labores, situándolas en una posición valorativa inferior en la estratificación simbólica laboral y, por tanto, se les retribuye con menor salario y beneficios (Honneth, 2006). 
Desde el punto de vista del proceso de regulación migratoria es clave considerar que la falta de reconocimiento oportuno por parte del Estado en materia migratoria les impide situarse en un lugar activo en la defensa de sus derechos laborales que les permita exigir un mayor reconocimiento, el que está presente en sus aspiraciones, pero por razones pragmáticas suspenden.

La estima social, por su parte, se vincula con el aporte que se percibe que estas mujeres hacen en sus trabajos, donde se destaca que la valoración manifestada por sus compañeros/as hacia ellas se relaciona con la posición de clase social y trayectoria profesional. Lo anterior confirma lo propuesto por Thayer (2012), quien afirma que en los empleos no calificados las migrantes llegan a ocupar empleos precarios, dentro de los cuales, incluso, se presenta una desigualdad que se intensifica cuando las características de las mujeres migrantes se intersectan (Crenshaw, 2013), jugando esta categoría un papel fundamental que evidencia que la experiencia de las mujeres está atravesada de manera simultánea por expresiones de racismo, sexismo y xenofobia que amplían las experiencias de injusticia.

La valoración cultural de capacidades de éxito demarca las profesiones (Honneth, 2006), poniendo de relieve que las trayectorias laborales de las mujeres migrantes que se desempeñan en trabajos masculinizados son valoradas, básicamente, porque se da socialmente más valor global, como totalidad, a un empleo masculinizado que a uno feminizado, lo que pone de manifiesto que el puesto de trabajo que ocupan en sí mismo es el que tiene mayor prestigio, y que sin embargo, lo que carece de menor prestigio son ellas mismas ejecutando dichas labores. Por tal motivo, hay quienes no consideran "normal" que una mujer (mal valorada en tanto su sexo) trabaje en un empleo al que le han sido asignadas características consideradas como masculinas, reproduciéndose así la injusticia a partir de una falta de reconocimiento de sus capacidades a propósito de su condición de género. Sin embargo, esto corrobora que la experiencia de cada migrante debe observarse con detenimiento, atendiendo a otras características de diferenciación que actúan complejizando el fenómeno de la falta de reconocimiento.

En lo que respecta a su autovaloración, sucede algo similar. Si bien ellas consideran que tienen un trabajo al que contribuyen con sus capacidades, dichas ideas están constantemente atravesadas por estereotipos e ideales de participación y aporte en el plano laboral, tradicionales desde una perspectiva de género, según las cuales asignan menor valor a la tarea misma que ellas realizan en un empleo que es socialmente más valorado cuando este es ejecutado por sus pares hombres. Frente a esta realidad, se observan las herramientas de resistencia que desarrollan las mujeres, las que se incrementan en la medida en que se ven expuestas a la falta de reconocimiento desde frentes distintos.

Considerando que el análisis se realizó en torno a mujeres migrantes de dos nacionalidades hispanohablantes (colombiana y venezolana), se abre la oportunidad de realizar nuevas investigaciones en torno a mujeres migrantes de otras nacionalidades, sobre todo no hispanohablantes, o incluso de nacionalidad chilena en otros países, lo que podría cualificar diversas vivencias cuando se intersectan características distintas. 
La experiencia de mujeres chilenas en los mismos empleos masculinizados, en los que la nacionalidad no se convierta en un eje de diferenciación, o la vivencia de mujeres de nacionalidades distintas, por ejemplo, haitianas, cuya "raza" o idioma podría incluso devenir en características de opresión más profunda que la nacionalidad o el género.

\section{Referencias bibliográficas}

Acosta, E. (2012). Valorar los cuidados al estudiar las migraciones: la crisis del trabajo de cuidado y la inmigración en Chile. En C. Stefoni (ed.), Mujeres inmigrantes en Chile ¿Mano de obra o trabajadoras con derechos? (pp. 193-228). Santiago: Ediciones Universidad Alberto Hurtado.

Acosta, E. (2013). Mujeres migrantes cuidadoras en flujos migratorios sur-sur y surnorte: expectativas, experiencias y valoraciones. Polis, 12(35), 35-62. DOI: $10.4067 /$ S0718-65682013000200003

Angelcos, N. e Ísola, E. (2017). Violencia y táctica en los procesos de integración de las mujeres a la minería del cobre en Chile. Psicoperspectivas, 16(2), 66-78. DOI: 10.5027/psicoperspectivas-vol16-issue2-fulltext-1019

Benería, L. (2006). Trabajo productivo/reproductivo, pobreza y políticas de conciliación. Nómadas, (24), 8-21.

Bourdieu, P. (2000). La dominación masculina. Barcelona: Anagrama.

Butler, J. (2011). Gender Trouble: Feminism and the Subversion of Identity. Nueva York: Routledge.

Canales, A. (2014). Migración femenina y reproducción social en los Estados Unidos. Inmigrantes latinas en los Estados Unidos. Revista Sociedad y Equidad, enero, (6), 160-188. DOI: 10.5354/0718-9990.2014.27267

Cárdenas, M. E. (inédita). Género y migración en mercados de trabajo masculinizados en Santiago de Chile: una mirada desde el reconocimiento. (Tesis para optar al grado de magíster en Trabajo Social). Pontificia Universidad Católica de Chile, Santiago.

Cárdenas, A., Correa, N. y Pardo, X. (2014). Segregación laboral y género: tendencias y desafíos relativos al mercado laboral de la salud y la educación en Chile. Polis, 13(38), 397-418. DOI: 10.4067/S0718-65682014000200018

Carrère, C. y Carrère, M. (2015). Inmigración femenina en Chile y mercado de trabajos sexualizados. La articulación entre racismo y sexismo a partir de la interseccionalidad. Polis Revista Latinoamericana, 14(42), 33-52. DOI: 10.4067/S0718-65682015000300003 
Cho, S., Crenshaw, K. W. y McCall, L. (2013). Toward a Field of Intersectionality Studies: Theory, Applications, and Praxis. Signs, 38(4), 785-810. DOI: $10.1086 / 669608$

Crenshaw, K. W. (1989). Demarginalizing the Intersection of Race and Sex: A Black Feminist Critique of Antidiscrimination Doctrine, Feminist Theory and Antiracist Politics. University of Chicago Legal Forum, 1989(1) artículo 8, 139167. Recuperado de http://chicagounbound.uchicago.edu/uclf/vol1989/iss1/8

Collins, P. y Bilge, S. (2016). Intersectionality. Malden: Polity Press.

Comunidad Mujer. (2016). Género, educación y trabajo: la brecha persistente. Primer estudio sobre la desigualdad de género en el ciclo de vida. Una revisión de los últimos 25 años. Chile. Informe GET. Recuperado de http://informeget.cl/wpcontent/uploads/2018/07/Informe-GET-2016.pdf

Cubillos, J. (2015). La importancia de la interseccionalidad para la investigación feminista. Oxímora, (7), 119-137. Recuperado de https://revistes.ub.edu/index. php/oximora/article/view/14502

Curiel, O. (2014). Construyendo metodologías feministas desde el feminismo decolonial. En: I. Mendia, M. Luxán, M. Legarreta, G. Guzmán, I. Zirion y J. Azpiazu (eds.), Reflexiones, herramientas y aplicaciones desde la investigación feminista (pp. 45-61). Bilbao: Hegoa.

Díaz, E. (2014). Mujeres en trabajos de hombres: segregación ocupacional y condiciones laborales en los sectores minería y construcción. Santiago: Gobierno de Chile, Dirección del Trabajo. Recuperado de http://www.dt.gob.cl/documentacion/1612/articles-103028_recurso_1.pdf

Espinoza, Y. (2016). De por qué es necesario un feminismo descolonial: diferenciación, dominación co-constitutiva de la modernidad occidental y el fin de la política de identidad. Solar, 12(1), 141-171. DOI: 10.20939/solar.2016.12.0109

Fraser, N. (2006). La justicia social en la era de la política de identidad: redistribución, reconocimiento y participación. En N. Fraser y A. Honneth (eds.), ¿Redistribución o reconocimiento?: un debate político filosófico (pp.19-91). Madrid: Morata.

Galaz, C., Becerra, M., Álvarez, C. y Hedrera, L. (2016). Intervención social con mujeres inmigradas en Chile: ¿asistencialismo y/o promoción social? Revista Rumbos TS, (14), 55-75. Recuperado de http://revistafacso.ucentral.cl/ index.php/rumbos/article/view/35

Galaz, C., Pavez, I., Álvarez, C. y Hedrera, L. (2019). Polivictimización y agencia de niños y niñas migrantes en Chile desde una mirada interseccional. Athenea Digital, 19(2), e2447. DOI: 10.5565/rev/athenea.2447 
Gissi, N., y Martínez, S. (2018). Trayectorias de género en la migración sur-sur de mujeres mexicanas calificadas en Santiago de Chile. Si Somos Americanos, 18(1), 83-118. DOI: 10.4067/S0719-09482018000100083

Honneth, A. (2006). Redistribución como reconocimiento: Respuesta a Nancy Fraser. En N. Fraser y A. Honneth, ¿Redistribución o reconocimiento?: Un debate político filosófico (pp. 92-150). Madrid: Morata.

Instituto Nacional de Estadísticas (INE). (2018). Características de la inmigración internacional en Chile, Censo 2017. Santiago: Autor. Recuperado de http://www.censo2017.cl/descargas/inmigracion/181123-documento-migracion. pdf

Instituto Nacional de Estadísticas (INE) y Departamento de Extranjería y Migración (DEM). (2020). Estimación de personas extranjeras residentes habituales en Chile al 31 de diciembre 2019. Informe Técnico. Estadísticas Migratorias, marzo, 1-27. Recuperado de https://www.ine.cl/docs/default-source/demografiay-migracion/publicaciones-y-anuarios/migración-internacional/estimaciónpoblación-extranjera-en-chile-2018/estimación-población-extranjera-en-chile2019-metodología.pdf?sfvrsn=5b145256_6

Jiménez, C., Rojas, P. y Troncoso, R. (2014). Género, trabajo y subjetividad: el lugar de la mujer en la minería. Persona y Sociedad, 28(3), 65-96. DOI: 10.11565/pys.v28i3.74

Leiva, S., Mansilla, M. A. y Comelin, A. (2017). Condiciones laborales de migrantes bolivianas que realizan trabajo de cuidado en Iquique. Si Somos Americanos, 17(1), 11-37. DOI: 10.4067/S0719-09482017000100011

Lobos, D. (2017). Migración y trabajo doméstico: las trabajadoras inmigrantes asociadas en el SINAINCAP en Santiago de Chile, 2012-2017. (Informe para optar al grado de licenciada en Historia). Facultad de Filosofía y Humanidades, Universidad de Chile, Santiago. Recuperado de http://repositorio.uchile.cl/ handle/2250/146880

Lugones, M. (2005). Multiculturalismo radical y feminismos de mujeres de color. Revista Internacional de Filosofía Política, (25), 61-76. Recuperado de https://www.redalyc.org/pdf/592/59202503.pdf

Martínez, J. y Orrego, C. (2016). Nuevas tendencias y dinámicas migratorias en América Latina y el Caribe. Serie Población y Desarrollo $N^{\circ}$ 114. Santiago: Naciones Unidas. Recuperado de https://www.cepal.org/es/publicaciones/39994nuevas-tendencias-dinamicas-migratorias-america-latina-caribe

Ministerio de Desarrollo Social. (2017). Casen 2017: Inmigrantes, síntesis de resultados. Santiago: Autor. Recuperado de http://observatorio.ministerio desarrollosocial.gob.cl/storage/docs/casen/2017/Resultados_Inmigrantes_casen_ 2017.pdf 
Monje, C. (2011). Metodología de la investigación cuantitativa y cualitativa, guía didáctica. Neiva: Universidad Surcolombiana.

Organización Internacional del Trabajo (OIT). (2017). Panorama laboral 2017 América Latina y El Caribe. Lima: OIT. Recuperado de https://www.ilo.org/wcmsp5/ groups/public/---americas/---ro-lima/documents/publication/wcms_613957.pdf

Organización Internacional para las Migraciones (OIM). (2018). Informe sobre las migraciones en el mundo 2018. Ginebra: OIM. Recuperado de https://publications.iom.int/system/files/pdf/wmr_2018_sp.pdf

Pateman, C. (1988). The Sexual Contract. Stanford, Cal.: Stanford University Press.

Pavez-Soto, I. y Galaz, C. (2018). Hijas e hijos de migrantes en Chile: derechos desde una perspectiva de inclusión social. Diálogo Andino, (57), 69-82. Recuperado de http://dialogoandino.cl/wp-content/uploads/2018/12/06-PAVEZ-Y-GALAZRDA57.pdf

Rojas, N. y Silva, C. (2016). La migración en Chile: breve reporte y caracterización. Informe OBIMID. Madrid: OBIMID. Recuperado de https://n9.cl/nfgh5

Piñeiro, D. (2011). Precariedad objetiva y subjetiva en el trabajo rural: nuevas evidencias. Revista de Ciencias Sociales, 24(28), 11-33. Recuperado de https://www.redalyc.org/pdf/4536/453644789002.pdf

Riquelme, G., López, A. y Olivares, V. E. (2014). Estudio empírico del trabajo calificado en Chile: productividad y remuneraciones. Ciencia y Trabajo, 16(50), 81-87. DOI: $10.4067 /$ S0718-24492014000200004

Salas, R. (2014). Las inmigrantes nicaragüenses en Costa Rica: migración, inserción social y procesos organizativos. Temas de Antropología y Migración, diciembre, (7), 7-30. Recuperado de http://www.migrantropologia.com.ar/images/stories/ PDF/Revista7/t07a01.pdf

Salinas, P. y Romaní, G. (2016). Hegemonía masculina, freno en equipos mixtos en la minería chilena. Revista Mexicana de Sociología, 78(3), 469-496. Recuperado de https://www.researchgate.net/publication/306012371_Hegemonia_Masculina _freno_en_equipos_mixtos_en_la_mineria_chilena

Scott, J. W. (1986). Gender: A Useful Category of Historical Analysis. The American Historical Review, 91(5), 1053-1075. DOI: 10.2307/1864376

Suárez-Cabrera, D. (2015). Nuevos migrantes, viejos racismos: los mapas parlantes y la niñez migrante en Chile. Revista Latinoamericana de Ciencias Sociales, Niñez y Juventud, 13(2), 627-643. DOI: 10.11600/1692715x.1325110414

Stefoni, C. (2014). Perspectiva transnacional en los estudios migratorios. Revisión del concepto y nuevos alcances para la investigación. En W. Imilan, A. Garcés, y D. Margarit (eds.), Poblaciones en movimiento, etnificación de la ciudad, redes e integración (pp. 41-65). Santiago: Ediciones Universidad Alberto Hurtado. 
Stefoni, C. (2018). Panorama de la migración internacional en América del Sur. Documento elaborado en el marco de la Reunión Regional Latinoamericana y Caribeña de Expertas y Expertos en Migración Internacional preparatoria del Pacto Mundial para una Migración Segura, Ordenada y Regular. Santiago: Naciones Unidas. Recuperado de https://repositorio.cepal.org/bitstream/ handle/11362/43584/1/S1800356_es.pdf

Stefoni, C. y Fernández, R. (2012). Mujeres inmigrantes en el trabajo doméstico. Entre el servilismo y los derechos. En C. Stefoni (ed.), Mujeres inmigrantes en Chile: ¿Mano de obra o trabajadoras con derechos? (pp. 43-72). Santiago: Ediciones Universidad Alberto Hurtado.

Stefoni, C., Leiva, S. y Bonhomme, M. (2017). Migración internacional y precariedad laboral. El caso de la industria de la construcción en Chile. REMHU: Revista Interdisciplinar da Mobilidade Humana, 25(49), 95-112. DOI: 10.1590/198085852503880004906

Stefoni, C. y Stang, F. (2017). La construcción del campo de estudio de las migraciones en Chile: notas de un ejercicio reflexivo y autocrítico. Íconos - Revista de Ciencias Sociales, (58), 109-129. DOI: 10.17141/iconos.58.2017.2477

Thayer, E. (2012). Trabajo y género: la condición social de inmigrante como referente para la definición de la identidad. En C. Stefoni, Mujeres inmigrantes en Chile: ¿Mano de obra o trabajadoras con derechos? (pp. 43-72). Santiago: Ediciones Universidad Alberto Hurtado.

Thayer, E. (2013). Expectativas de reconocimiento y estrategias de incorporación: la construcción de trayectorias degradadas en migrantes latinoamericanos residentes en la Región Metropolitana de Santiago. Polis, Revista Latinoamericana, 12(35), 259-285. DOI: 10.4067/S0718-65682013000200012

Tijoux, M. E. y Palominos, S. (2015). Aproximaciones teóricas para el estudio de procesos de racialización y sexualización en los fenómenos migratorios de Chile. Polis, Revista Latinoamericana, 14(42), 247-275. Recuperado de https://scielo.conicyt.c1/pdf/polis/v14n42/art_12.pdf

Troncoso, L., Follegati, L. y Stutzin, V. (2019). Más allá de una educación no sexista: aportes de pedagogías feministas interseccionales. Pensamiento Educativo. Revista de Investigación Educacional Latinoamericana, 56(1), 1-15. DOI: 10.7764/PEL.56.1.2019.1

Troncoso, L., Galaz, C. y Álvarez, C. (2017). Las producciones narrativas como metodología de investigación feminista en Psicología Social Crítica: Tensiones y desafíos. Psicoperspectivas, 16(2), 20-32. DOI: 10.5027/psicoperspectivasvol16-issue2-fulltext-956

Trpin, V (2007). Identidades en movimiento: familias chilenas en la fruticultura del Alto Valle de Río Negro, Argentina. Cadernos Pagu, jul.-dic., (29), 227-255. DOI: $10.1590 / \mathrm{S} 0104-83332007000200010$ 
Valdés, G., y Meller, P. (2014). Análisis de los diferenciales salariales según género entre profesionales chilenos. Interciencia, 39(12), 836-842. Recuperado de https://www.interciencia.net/wp-content/uploads/2017/11/836-Valdes-7.pdf

Vidal, M. (2017). La segregación horizontal por género y sus consecuencias en la ocupación masculinizada de mecánico/a en el subsector de reparación de vehículos en España. Laboreal, 13(1), 1-22. DOI: 10.4000/laboreal.1796

Viveros, M. (2016). La interseccionalidad: una aproximación situada a la dominación. Debate feminista, 52, october, 1-17. DOI: 10.1016/j.df.2016.09.005 